\title{
"BULLYING" EN LAS FACULTADES DE MEDICINA COLOMBIANAS, MITO O REALIDAD
}

\author{
Olga Lucía Paredes, Ps. M.Sc ${ }^{1}$, Pablo-Alfonso Sanabria-Ferrand Ps. M.Sc ${ }^{1 *}$, \\ Luis Artemo González-Quevedo, Ps ${ }^{1}$ y Sandra Patricia Moreno Rehalpe, MD. M.Sc ${ }^{1}$ \\ ${ }^{1}$ Grupo de Investigación Salud y Comportamiento, Facultad de Medicina, \\ Universidad Militar Nueva Granada, Bogotá.
}

\begin{abstract}
Resumen
Tradicionalmente, los estudios de "Bullying", también conocido como intimidación o matoneo, se han concentrado en la enseñanza primaria y secundaria, pero pocos han dirigido su interés al ámbito de la educación superior y en menor número en los programas de salud. En este artículo se presenta los resultados de un estudio diseñado para identificar y caracterizar la presencia del "bullying" en los estudiantes de pregrado de 22 facultades de medicina en Colombia, como un aporte para la generación de políticas de calidad de la educación médica en el país. Se utilizó un diseño transversal de tipo descriptivo-comparativo, con una muestra probabilística de 1.500 estudiantes de pregrado de los primeros diez semestres. La prevalencia general encontrada de "bullying" fue del 19,68\% (IC 95\%= $17,5-22,32)$. Las zonas de mayor prevalencia fueron la costa $(28,01 \%$, IC95\% $=22,53-33,48)$, la zona oriental $(26,29$, IC95\% $=21,38-31,19)$ y la zona central $(23,56$, IC95\% = 17,40-29,71). La fuente más frecuente de matoneo fueron los docentes y los compañeros, siendo más común la intimidación por humillación y de tipo verbal. No se encontraron diferencias significativas entre los diferentes tipos de universidades, sexos ni semestres cursados. Se evidencia, por tanto, que el "bullying" no es un mito sino una realidad, y que debe ser un tema de preocupación para las facultades y hospitales universitarios, en la medida que podría estar afectando la dinámica de relaciones interpersonales, la permanencia en la carrera, el rendimiento académico, la productividad e incluso la salud de los médicos y de sus pacientes, siendo este último un aspecto importante por estudiar. Los resultados obtenidos hacen pensar que es urgente desarrollar programas de intervención para la prevención y manejo de comportamientos que ocasionan "bullying" en las facultades de medicina.
\end{abstract}

Palabras clave: estudiantes de medicina, intimidación, violencia.

\section{BULLYING IN COLOMBIAN MEDICINE FALCULTIES, MYTH OR REALITY}

\begin{abstract}
Research on bullying or intimidation have mainly focus in the elementary and secondary education, while few have been conducted on superior education and even less in the health programs. This article presents the results of a study designed to identify and to characterise the presence of bullying in undergraduate students of 22 medicine faculties of Colombia, as a contribution for the generation of quality policies underproducate for the medical education in this country. A transversal, descriptive and comparative design was used, with a probabilistic sample of 1,500 undergraduate students of the first ten semesters. The general prevalence found for bullyng was of 19,68\% (IC 95\% $=17,5-22,32$ ). The areas of higher prevalence were the coast $(28,01 \%$, IC $95 \%=22,53-33,48)$, the eastern zone
\end{abstract}

* Correspondencia: Pablo Alfonso Sanabria pablo.sanabria@unimilitar.edu.co, Dirección Postal: Tr. 5 No 49-00, Facultad de Medicina, Universidad Militar Nueva Granada, Bogotá, Colombia

Recibido: Noviembre 3 de 2010 Aprobado: Diciembre 15 de 2010 
$(26,29$, IC95\% $=21,38-31,19)$ and the central zone $(23,56$, IC95\% $=17,40-29,71)$. The bullies were lecturers and class mates and the most common form was intimidation by humiliation of verbal type. Non significant differences were found among different types of universities, the gender or coursed semesters. Evidences are presented supporting that bullying is a reality not a myth, and hence it must be a matter of attention for faculties and hospitals, since it is affecting the dynamics of interpersonal relationship, the permanence in the program, the academic performance, the productivity and even the health of the practitioners and their patients. For all these reasons, it becomes imperative to develop intervention programs in preventing and handling of bullying behaviours in the medicine faculties.

Key words: medical students, bullying, violence

\title{
“BULLYING” NAS ESCOLAS COLOMBIANAS DE MEDICINA, MITO OU REALIDADE
}

\section{Resumo}

\begin{abstract}
Tradicionalmente, os estudos de "bullying", também conhecido como intimidação ou assédio moral, centraram-se na educação primária e secundária, mas poucos se voltaram seu interesse para o campo do ensino superior e menos para os programas de saúde. Este artigo apresenta os resultados de um estudo destinado a identificar e caracterizar a presença do bullying em estudantes universitários de 22 faculdades de medicina na Colômbia, como uma contribuiçao para o desenvolvimento de políticas de qualidade da educação médica no país. Utilizou-se um estudo de corte transversal, descritivo-comparativo, com uma amostra probabilística de 1.500 alunos de graduação nos primeiros dez semestres. Foi encontrada uma prevalência global de bullying foi $19,68 \%(95 \% \mathrm{CI}=17,5-22,32)$. As áreas de maior prevalência foram o litoral $(28,01 \%$, IC $95 \%=22,53-33,48)$, leste $(26,29$, IC $95 \%=21,38-31,19)$ e centrais $(23,56$, IC $95 \%=17,40-29,71)$. A fonte mais comum de assédio moral foram os professores e colegas, sendo mais comum a intimidação pela humilhação é de tipo verbal. Não houve diferenças significativas entre os diferentes tipos de universidades, sexos e semestres tomadas. É evidente, portanto, que o "bullying" não é um mito, mas uma realidade, e deve ser uma preocupação para os faculdades e hospitais universitários, como pode estar afetando a dinâmica das relações interpessoais, a permanencia na faculdade, o desempenho acadêmico, as produtividade e até mesmo a saúde dos médicos e seus pacientes, sendo esto último um aspecto importante para estudo. Os resultados sugerem que é urgente o desenvolvimento de programas de intervenção para a prevenção e abordagem de comportamentos que provocam "bullying" nas escolas de medicina.
\end{abstract}

Palavras-chave: estudantes de medicina, bullying, violencia

\section{Introducción}

En los últimos años la humanización de la atención en salud ha sido un motivo de preocupación global, así como el interminable número de demandas por negligencia y errores médicos, las quejas por la calidad de la asistencia, el distanciamiento de la relación médico-paciente y el funcionamiento del sistema general de salud, que siguen sumándose a las problemáticas que deben asumir los médicos. Las innovaciones más recientes en cuanto a la tecnología médica a nivel mundial y las políticas regionales del sistema de salud, demandan de los profesionales el mejor desempeño de su ejercicio, para mejorar la eficiencia en las instituciones de salud y mantenerse en el mercado laboral. Estos cambios contextuales, ha diluido lo que se consideraba fundamental en la profesión médica, como es el cuidado de la salud de las personas.

Además de dichas preocupaciones, existe una nueva tendencia a estudiar también los aspectos relacionados con el bienestar de los profesionales de la salud en medio de su labor profesional (1). Factores como el burnout o desgaste profesional, la depresión, la ansiedad, el estrés y el suicidio (2), han sido reconocidos como factores importantes que influyen en la satisfacción de los profesionales, en sus prácticas médicas y en la rotación de sus empleos, entre otras cosas (3-5). En los 
últimos años, la violencia ha despertado un especial interés en los sitios de trabajo y de mayor importancia pero con menos desarrollo, la violencia interpersonal ejercida por pares en las escuelas de medicina.

La violencia ejercida por iguales, conocida como matoneo o intimidación en castellano, ocurre cuando una persona es agredida o expuesta a acciones negativas intencionales por parte de sus compañeros, sumado a un desequilibrio de poder, generando efectos nocivos en la calidad de vida y bienestar de la víctima y en los procesos del contexto en que se suscita (6).

El Instituto Nacional de Medicina Legal de Bogotá, Colombia, en el año 2007 reportó 34.258 lesiones por violencia interpersonal de las cuales, 965 casos señalan a su compañero como agresores directos; para el 2009 la cifra aumentó a 37.977 casos (7). La violencia entre pares o compañeros es un problema más serio y mucho más común de lo que la gente podría considerar, que produce efectos secundarios muy dañinos y que en muchos casos permanecen ocultos, convirtiéndose en un factor de riesgo para la salud de los profesionales y en un problema para su adecuada formación.

\section{¿Qué es el "bullying"?}

Inicialmente, el término "mobbing" fue utilizado por el etólogo Konrad Lorenz para describir un comportamiento que define un ataque de grupo de animales pequeños amenazando a uno más grande para su supervivencia (8). Años más tarde fue retomado por el médico Heinemann (1972) para hacer referencia a comportamientos de agresión entre niños (9). Posteriormente, el término, migró a "bullying" con las investigaciones realizadas en los países nórdicos y en Gran Bretaña a principio de los 70s (10).

El concepto del "bullying" resume actos de intimidación entre pares (iguales) consistentes en maltrato a través de acciones como diferentes tipos de maltrato y exclusiones sociales de forma directa o indirecta. Ejemplos de maltrato físico indirecto podrían se: esconder, robar o romper cosas de alguien, y directo: pegar, empujones, patadas, agresiones con objetos, amenazar con armas. De maltrato verbal indirecto serían: hablar mal de alguien, difundir rumores falsos, menosprecios en público, resaltar defectos físicos, y directo: insultar, burlarse, utilizar apodos. Relacionados con exclusión social indirecta: ignorar, rebajar, tratar como un objeto, y directa: excluir, no dejar participar a alguien en una actividad. Maltrato psicológico indirecto: acecho, minar la autoestima del sujeto y fomentar su sensación de temor y directo: gestos de asco, desprecio o agresividad dirigidos contra la víctima. Maltrato mixto (físico y verbal): amenazar para intimidar, obligar a hacer cosas, chantaje, acosar sexualmente y otros (11). En los últimos años se ha incluido el "cyberbullying", entendido como el acoso a través de medio tecnológicos interactivos (correos electrónicos, foros de Internet, mensajes de texto, redes sociales entre otros (12).

Yildirim y Yildirim (13) mencionan que a este despliegue de actitudes antagonistas en los contextos de estudio y trabajo se les ha dado diversos nombres y se han aplicado indistintamente en conceptos como: el terror psicológico, acoso, abuso laboral, "bullying", trauma laboral, chivo expiatorio y "mobbing" (14-20). Aparentemente estos términos pueden parecer iguales pero existen diferencias importantes especialmente en cuanto a quién se dirigen y al objetivo que pretenden conseguir. Puede darse de superiores a subordinados o entre pares, refiriéndose en éste último caso, a la ausencia de una jerarquía estipulada entre las dos partes. Estas características son por ejemplo, aquellas que permite distinguir el matoneo de un conflicto, pues este último no genera desequilibrio de fuerzas, no ocurre reiteradamente en el tiempo, se desarrolla de una forma abierta y puede conllevar a resultados positivos.

Los actores del "bullying" tienden por lo general, a presentar comportamientos agresivos y violentos, sobresalen del grupo por alguna característica, generalmente fuerza física o poder de intimidación. La víctima por su parte se evidencia del grupo por su dificultad para relacionarse o su inhabilidad para reaccionar, alguna característica física que resalta e incluso por su orientación religiosa, sexual y género. Los testigos se consideran protagonistas fundamentales en el "bullying" dado que pueden apoyar con su "pasividad" las acciones del victimario, lo que los convierte en parte activa del matoneo.

Las consecuencias de la intimidación no pueden ser ignoradas, ya que comprenden efectos para el individuo y para la organización, a corto y largo plazo. Entre las repercusiones para la víctima pueden encontrase la baja autoestima, sentimientos de culpa, falta de poder, desamparo, desesperanza y el aislamiento tanto de la tarea que se desempeña como del grupo de compañeros. Entre los efectos para la salud están cefaleas, migraña, nauseas, erupciones de piel, aumento de la 
tensión arterial, estrés, ansiedad, depresión, ataques de pánico, trastornos de sueño y de alimentación, abuso de drogas o de alcohol, estrés post traumático $e$ intentos de suicidio (21-22).

Su efecto sobre el rendimiento académico también es un aspecto importante a considerar. Se ha evidenciado que estudiantes que han sido matoneados tienen dificultades con la atención y concentración, y gastan su tiempo activo defendiéndose de otros, buscando apoyo social y pensando acerca de la situación, hechos que entorpecen el proceso de enseñanza-aprendizaje $(21,22)$. En el ámbito organizacional, se ha reportado desmotivación, baja eficacia, ausentismo, "burnout", conflictos y degradación del clima laboral, baja moral, miedo, irritabilidad, y enfermedades por estrés. También hay rotación continúa de estudiantes o trabajadores, demandas, intención de abandono de la carrera y otras actuaciones que tienen implicaciones financieras (23-25).

\section{"Bullying" en las escuelas de medicina}

Las estadísticas mundiales del "bullying" en colegios señalan una prevalencia que van de un 15 a un $68 \%$. En el País Vasco, por ejemplo, ha alcanzado un 15\% de presencia del matoneo en las escuelas, mientras que en Colombia se habla de un $30 \%$ de jóvenes intimidados (26), cifra bastante similar a la de Estados Unidos (27).

Se han reportado los resultados de investigaciones realizadas en otros países sobre este tema, en diferentes áreas de formación de instituciones educación superior tales como enfermería $(28,29)$, gastronomía (30) y humanidades y tecnología (31), al igual que en la milicia (32) y la política (33). La intimidación ocurre en diferentes instituciones, aunque las tasas tienden a ser más altas en las organizaciones relacionadas con la salud y dentro de las instituciones de educación superior, se observa más en las facultades de medicina que en las otras (34). En Colombia, en el año 2009, Hoyos y col. (35) desarrollaron un trabajo de investigación, en donde se evidencio la presencia de maltrato entre iguales en universitarios que cursaban del segundo a los penúltimos semestres de distintos programas académicos ofrecidos por una universidad privada de la ciudad de Barranquilla, aunque este no específica si existió o no una muestra de estudiantes de medicina.

Wood asegura que el "bullying" se constituye en uno de los problemas más reconocidos pero menos resueltos durante la formación académica (36). El comportamiento de intimidación se ha determinado como un efecto negativo importante en la escogencia de carrera, la permanencia en la profesión y el desempeño profesional. Por su parte, Quine reporta los resultados de una investigación realizada en Inglaterra con residentes de especialidades médicas, en donde encontró niveles de "bullying" de un $37 \%$ en los médicos de los primeros niveles de formación, y un $84 \%$ de todos los estudiantes experimentaron por lo menos un comportamiento de "bullying" durante sus años de formación. Es de anotar, que esta investigación reporta que las personas de raza negra o asiáticos y las mujeres eran quienes sufrían más intimidación (37).

El grupo de Frank y col. realizaron una investigación para indagar el porcentaje de acoso y subestimación que ejercen residentes, becarios, docentes preclínicos, docentes clínicos y pacientes sobre los estudiantes de primer nivel, los estudiantes que estaban iniciando turnos clínicos y los estudiantes más avanzados. Encontraron que en los estudiantes existe un $42 \%$ de acoso y $84 \%$ de subestimación. En cuanto a los agresores, se encontró que un $27 \%$ acosaban y en un $71 \%$ subestimaban. Por otra parte, en los docentes preclínicos, el acoso fue del 9\%, y la subestimación del 29\%; los docentes clínicos eran responsables de un $21 \%$ de acoso y un $63 \%$ de subestimación. En este estudio no se evidenciaron diferencias en severidad del acoso por pertenencia a una raza o religión, pero sí por género, siendo las mujeres quienes evidenciaron mayores tasas de estrés, depresión y suicidio, abuso de alcohol y consideración de que la facultad no se interesaba por ellas. Los alumnos acosados o subestimados eran menos felices y se encontraban menos satisfechos de convertirse en médicos profesionales (34).

Los resultados obtenidos en un trabajo realizado por Rautio y col. coinciden con los anteriores datos. En su estudio encontró que la mitad de los estudiantes médicos han experimentado alguna forma de maltrato por parte del equipo médico durante sus años de estudios, más comúnmente acciones como humillación (40\%), recalcar aspectos negativos o despectivos (34\%), gritar $(23 \%)$, acoso sexual o por género (17\%) y tareas asignadas como castigo (13\%) (31). También se muestra un porcentaje mayor de maltrato en los estudiantes de medicina $(32 \%)$ que en los estudiantes de otras facultades, como Humanidades, Educación y Ciencia y Tecnología. Hicks por su parte encontró cifras más altas, el 91,7\% de los estudiantes encuestados habían 
sufrido, por lo menos, un episodio de abuso por parte de profesores y compañeros. El más común fue el abuso verbal $(85,4 \%)$, seguido por el psicológico $(79,9 \%)$, el sexual $(26,4 \%)$ y el físico $(23,6 \%)$. Los alumnos consideraban que esta situación había tenido efecto en su salud mental, su vida social y la imagen que ellos tenían de los médicos. Un 17\% habían considerado abandonar el estudio por la experiencia relacionada con los eventos mencionados (38).

Autores como Kassebaum y Cutler, a partir de este tipo de estudios, plantean que la cultura de abuso está considerada como parte de la misma formación del médico (39), Además afirman, que los estudiantes de medicina lo consideran como un comportamiento normal e incluso que son útiles como experiencia de aprendizaje, mientras que los estudiantes de carreras diferentes, no tienen esa percepción, por lo tanto tienen mayor posibilidad de reportar incidentes de acoso. Además del anterior planteamiento, se suma el poco respaldo a los estudiantes por parte de las instituciones de formación médica, lo que podría estar validando este tipo de regla de formación.

Además, es relevante resaltar la irónica tendencia que tienen de los médicos de estar poco interesados en su propia salud; desconocer el verdadero impacto que tienen los estilos de vida en ellos y en sus pacientes, en dar poca relevancia a la promoción de su salud y la falta de búsqueda apoyo para resolver sus problemas emocionales (40-43). Podría pensarse que dar y recibir abuso es considerado un proceso de socialización necesario para obtener la fortaleza que requiere ser médico.

Por otra parte, existen organizaciones que permiten que ocurran comportamientos de violencia e intimidación. Estas organizaciones se caracterizan por ejemplo, por dinámicas de largas horas de trabajo, con pocas posibilidades de sociabilidad y en las cuales en las horas pico, aumentan la presión porque las necesidades de los usuarios deben ser satisfechas inmediatamente. Carecen de servicios de apoyo y bienestar para sus profesionales, con un ausente sistema de recompensas, justicia y control, y en ocasiones, de valores.

Pero no son únicamente las condiciones propias de la institución o la función las que toleran que se presente la intimidación; los procesos de socialización también legitiman la violencia interpersonal. Haidet y Stein sugieren que los métodos de socialización de éste tipo pueden ser similares a los de un culto, originándose en un ambiente controlado, de autoridad no cuestionada y la utilización de "juegos" como reglas (44). Uno de éstos métodos es el del currículo oculto, definido como "el proceso por el cual una persona aprende los valores, normas y comportamientos requeridos que permiten participar como un miembro de la organización" (45). Este grupo de influencias incluyen reglas implícitas para sobrevivir y aspectos y rituales que se dan por sentados. El estudio concluye que en las facultades de medicina, en las que pueden existir instrucciones caóticas y enseñanza con humillación, los estudiantes reportan modelos positivos y efectivos, maestros accesibles, pero también una atmósfera de jerarquía y competencia. Fekkes, Pijpers, y VerlooveVanhorick le atribuyen un papel protagónico a las autoridades en estos escenarios (46), colaborando con la transmisión de actitudes y acciones que legitiman la intimidación a una persona específica que ellos también han "señalado", al exigirle continuamente un desempeño mayor o bien llamándolo con algún adjetivo descalificativo.

En conclusión el estudio de estas formas de educación pueden traer grandes aportes al abordaje de la problemática de la violencia dentro de las escuelas $e$ instituciones médicas, de una forma más efectiva, permitiendo conocer de fuentes primarias la dinámica de las relaciones entre compañeros y también de docente-estudiante. El objetivo de este trabajo fue Identificar y caracterizar la presencia del "Bullying" en los estudiantes de pregrado en las Facultades de Medicina del país como un aporte para la generación de políticas de calidad de la educación médica en Colombia.

\section{Metodología}

Se utilizó un diseño de tipo descriptivo-comparativo.

\section{Población y muestra:}

Se tomó como población de referencia, todas las facultades inscritas a la Asociación Colombiana de Facultades de Medicina (Ascofame) que respondieron a la convocatoria hecha para participar en el estudio, por lo que el universo lo constituyeron los estudiantes de pregrado de las facultades de medicina de los semestres II al X de 23 universidades del todo el país. Estos fueron seleccionados mediante un diseño de muestreo aleatorio, bietápica de conglomerados; en 
la primera etapa se seleccionaron semestres y en la segunda se seleccionaron estudiantes. La muestra la constituyó un total de 1.500 estudiantes distribuidos proporcionalmente en tres regiones en todo el país (1. Central y Antioquia y viejo Caldas; 2 . Costa atlántica y 3. Suroccidente y Oriente).

\section{Instrumentos}

Tomando como base la escala "Workplace bullying in junior doctors questionnaire" de la Dra. Lyn Quinne, del "Centre for Research in Health Behaviour", Universidad de Kent, Inglaterra (47), se diseñó un instrumento de 58 preguntas que evalúa el "Bullying" de compañeros, docentes, residentes y especialistas no docentes, de tipo: físico, verbal, rechazo y burla. La escala también evalúa las actitudes de los estudiantes hacia este tipo de prácticas, la intención de los estudiantes para impedirla y la práctica de la misma. Inicialmente en un ensayo piloto, el instrumento fue piloteado con 40 estudiantes de medicina de distintos semestres y la adaptación final del mismo se realizó con 100 estudiantes adicionales.

\section{Procedimiento}

Se establecieron los contactos institucionales a través de una comunicación dirigida a los decanos de las distintas facultades inscritas a Ascofame, en donde se les solicitó su participación voluntaria al estudio y la el número de estudiantes matriculados en el programa de pregrado. La delimitación del marco muestral se construyó con las facultades que respondieron al comunicado (80\%). La aplicación de los cuestionarios se realizó de manera grupal y anónima en cada facultad y su diligenciamiento se realizó posterior a la firma del consentimiento informado, en sesiones de una hora aproximadamente.

\section{Resultados}

Es necesario aclarar que si bien el "bullying" supone una relación de poder dialéctica entre víctima y victimario que se encuentran bajo la misma condición (de iguales), algunos autores asumen que una modalidad de la intimidación es la que se puede denominar: acoso docente, escondida en muchas ocasiones por ese ejercicio de autoridad (48). El cuestionario en el cual se basó el instrumento aplicado en la presente investigación indaga sobre el matoneo, asumiendo que algunos de los comportamientos de los supervisores docentes también se contemplan como intimidación, dado probablemente por el ambiente de trabajo que abarca la función de un estudiante médico en sus últimos años de estudio especialmente (47). Para efectos de los resultados no se resaltará la diferencia en los porcentajes cuando se hable de cada uno de estos grupos.

Los porcentajes encontrados de las diferentes conductas de "bullying" se muestran en la gráfica 1 . Se observa que la conducta de intimidación más frecuente es la humillación de los docentes $(9 \%)$ seguida del verbal por compañeros. Aunque el porcentaje es muy bajo, es importante anotar la aparición del "ciberbullying".

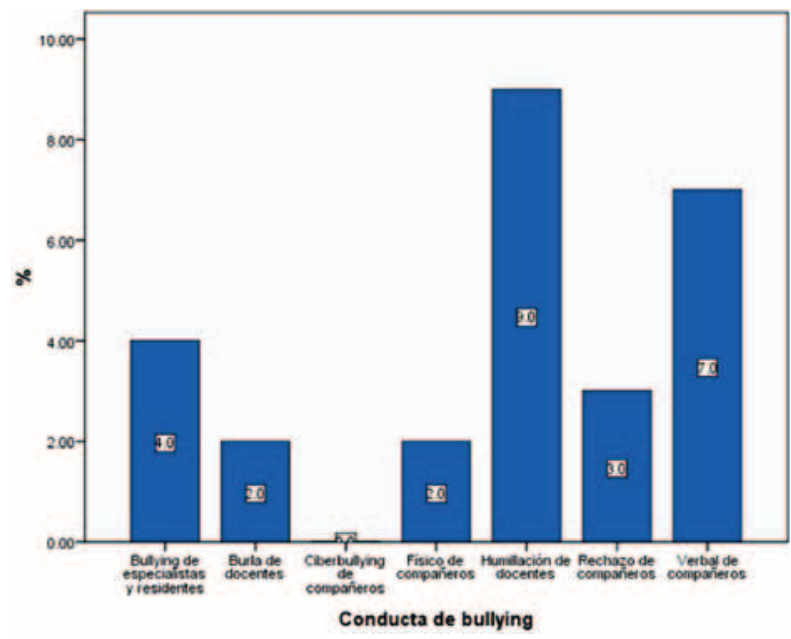

Gráfica 1. Porcentaje encontrado de las conductas de "bullying"

Al comparar los valores de "Bullying" en las diferentes regiones y el origen (compañeros o docentes), se observa que el porcentaje más alto de intimidación se observa en la Costa (28\%) seguida de Oriente (26\%) y Centro (26,3\%), mientras que los más bajos se encuentran en el Occidente $(14,8 \%$ ) y en la región de Antioquia y viejo Caldas (11\%).

La Costa obtuvo el porcentaje más alto en todos los tipos de intimidación, siendo el rechazo el más alto. Le sigue la región Oriente en donde se observa un nivel de intimidación de tipo físico, verbal y "ciberbullying". Se encuentra también un alto porcentaje de matoneo de docentes y compañeros pero bajo en el de especialistas y residentes. En la región occidental, los porcentajes obtenidos fueron de los más bajos para casi todos los indicadores evaluados excepto para el "bullying" 
ejercido por especialistas y residentes en donde los porcentajes encontrados fueron similares a los de la Costa. Finalmente, al comparar los porcentajes encontrados en todas las regiones, los de Antioquia son los más bajos y no se observó "cyberbullying". En esa misma región, entre todos los tipos de intimidación, la ejercida por compañeros, residentes y especialistas presentaba los valores más bajos (Tabla 1).

Tabla 1. Porcentaje de "Bullying" por compañeros y docentes en las diferentes regiones del país.

\begin{tabular}{|c|c|c|c|}
\hline & COMPAÑEROS & DOCENTES & TOTAL \\
\hline Antioquia & 5,0 & 5,5 & $\begin{array}{c}11,0 \\
\text { IC95\% }(6,54-15,52)\end{array}$ \\
\hline Central & 11,8 & 14,4 & $\begin{array}{c}23,6 \\
\text { IC95\% }(17,40-29,71)\end{array}$ \\
\hline Costa & 15,0 & 14,9 & $\begin{array}{c}28,0 \\
\text { IC95\% }(22,53-33,48)\end{array}$ \\
\hline $\begin{array}{l}\text { Occi- } \\
\text { dente }\end{array}$ & 6,1 & 5,3 & $\begin{array}{c}14,8 \\
\text { IC95\% }(9,30-20,39)\end{array}$ \\
\hline Oriente & 17,3 & 16,0 & $\begin{array}{c}26,3 \\
\text { IC95\% }(21,38-31,19)\end{array}$ \\
\hline
\end{tabular}

En el "bullying" por los docentes no se encontró diferencia entre el ejercido por hombres del ejercido por mujeres, mientras que en la intimidación por compañeros si se observó diferencia entre los sexos (Tabla 2). Con respecto a los niveles de matoneo y el tipo de universidad se observó una tendencia significativa a encontrarse valores de intimidación más alto en las Universidades públicas (Tabla 2).

Tabla 2. Porcentaje de "bullying" por compañeros y docentes de acuerdo al sexo del encuestado y al tipo de universidad

\begin{tabular}{lccc}
\hline & COMPAÑEROS & DOCENTES & TOTAL \\
\hline Masculino & 12,3 & 11,8 & 22,5 \\
Femenino & 8,7 & 10,1 & 18,2 \\
Pública & 12,4 & 11,7 & 23,4 \\
Privada & 9,0 & 10,2 & 18,0 \\
\hline
\end{tabular}

Con relación a las conductas que tuvieron los estudiantes frente al "bullying", se observó que la más frecuente fue ignorar lo que esta ocurriendo (66\%), seguida de contarlo a los amigos o compañeros. Las conductas de reportar a autoridades lo que estaba pasando fue apenas del 7\% (Tabla 3). Con respecto a estas conductas, no se observan diferencias respecto a la zona geográfica o al sexo de los alumnos.

Tabla 3. Porcentaje encontrado de diferentes conductas frente al "bullying"

\begin{tabular}{lc}
\hline \multicolumn{1}{c}{ Conductas } & $\mathbf{\%}$ \\
\hline Ignorar la intimidación & $65,8 \%$ \\
Solicitar cambio de curso o rotación & $3,6 \%$ \\
Confrontar la persona & $42,1 \%$ \\
Amenazar con contárselo a otros & $1,2 \%$ \\
Contárselo a algún amigo o compañero & $52,8 \%$ \\
Reportar a alguna autoridad académica o administrativa & $6,5 \%$ \\
Presentar la queja ante las instancias correspondientes & $7,2 \%$ \\
Otra acción & $4,9 \%$ \\
\hline
\end{tabular}

Otros de los aspectos indagados fue la percepción que tienen los estudiantes de porque las personas son objeto de "bullying". Se encontró que la mayoría de los estudiantes $(72 \%)$ creen que es debido a la forma de pensar, seguido de la apariencia física y la condición de ser hombre o mujer. No se observaron diferencias en los porcentajes por zonas geográficas. En cuanto al sexo de los estudiantes, se encontró que la mayoría de las mujeres, piensan que la condición de ser mujer y la apariencia física induce mas fácilmente ser objeto de intimidación; en cambio los hombres creen que es la preferencia sexual (Tabla 4).

Tabla 4. Porcentaje del tipo de condición que induce al "bullying".

\begin{tabular}{lc}
\hline \multicolumn{1}{c}{ Condiciones } & \% \\
\hline Ser mujer u hombre & $21,5 \%$ \\
Apariencia física & $28,4 \%$ \\
Orientación sexuales & $4,6 \%$ \\
Forma de pensar & $71,5 \%$ \\
\hline
\end{tabular}

En relación con la norma subjetiva, cuyos puntajes altos hacen referencia a que el "bullying" se percibe como una práctica esperada dentro del contexto estudiantil en medicina, se encontró en general que más o menos dos a tres de cada diez estudiantes consideran esta práctica como un comportamiento esperable y aceptable dentro del grupo. No se observan diferencias significativas en función del semestre académico, género o zona geográfica (Gráfica 5). 
Tabla 5. Porcentaje del tipo norma subjetiva hacia el "bullying".

\begin{tabular}{|c|c|c|c|c|c|}
\hline & \multirow{3}{*}{$\begin{array}{l}\text { Variables } \\
\text { Menor de V }\end{array}$} & \multicolumn{4}{|c|}{ Puntaje de Norma subjetiva } \\
\hline & & \multicolumn{2}{|c|}{ BAJO } & \multicolumn{2}{|c|}{ ALTO } \\
\hline \multirow{2}{*}{ Semestre } & & 725 & $76,8 \%$ & 219 & $23,2 \%$ \\
\hline & De VI en adelante & 498 & $74,8 \%$ & 168 & $25,2 \%$ \\
\hline \multirow{2}{*}{ Sexo } & Masculino & 469 & $72,3 \%$ & 180 & $27,7 \%$ \\
\hline & Femenino & 750 & $78,2 \%$ & 209 & $21,8 \%$ \\
\hline \multirow{5}{*}{ Zona geográfica } & Antioquia y viejo caldas & 166 & $78,3 \%$ & 46 & $21,7 \%$ \\
\hline & Central & 438 & $77,7 \%$ & 126 & $22,3 \%$ \\
\hline & Costa & 202 & $72,1 \%$ & 78 & $27,9 \%$ \\
\hline & Occidente & 193 & $76,3 \%$ & 60 & $23,7 \%$ \\
\hline & Oriente & 234 & $74,3 \%$ & 81 & $25,7 \%$ \\
\hline
\end{tabular}

Tabla 6. Porcentaje de intención de reaccionar contra el "bullying".

\begin{tabular}{llcccc}
\hline & & \multicolumn{2}{c}{ Intención } \\
\multirow{2}{*}{ Semestre } & Menor de V & \multicolumn{2}{c}{ BAJO } & \multicolumn{2}{c}{ ALTO } \\
& De VI en adelante & 30 & $3,2 \%$ & 913 & $96,8 \%$ \\
\multirow{2}{*}{ Sexo } & Masculino & 18 & $2,7 \%$ & 653 & $97,3 \%$ \\
\hline \multirow{3}{*}{ Zona geográfica } & Femenino & 25 & $3,8 \%$ & 626 & $96,2 \%$ \\
& Antioquia y viejo caldas & 25 & $2,6 \%$ & 939 & $97,4 \%$ \\
\hline & Central & 4 & $1,9 \%$ & 209 & $98,1 \%$ \\
& Costa & 19 & $3,4 \%$ & 541 & $96,6 \%$ \\
& Occidente & 7 & $2,5 \%$ & 270 & $97,5 \%$ \\
& Oriente & 11 & $4,3 \%$ & 244 & $95,7 \%$ \\
\hline
\end{tabular}

Tabla 7. Porcentaje de actitudes contra el "bullying".

\begin{tabular}{llcccc}
\hline & & \multicolumn{2}{c}{ Actitudes } \\
& & \multicolumn{2}{c}{ BAJO } & \multicolumn{2}{c}{ ALTO } \\
\hline \multirow{2}{*}{ Semestre } & Menor de V & 816 & $86,6 \%$ & 126 & $13,4 \%$ \\
& De VI en adelante & 602 & $90,1 \%$ & 66 & $9,9 \%$ \\
\hline \multirow{2}{*}{ Sexo } & Masculino & 516 & $79,5 \%$ & 133 & $20,5 \%$ \\
& Femenino & 901 & $93,8 \%$ & 60 & $6,2 \%$ \\
\hline \multirow{3}{*}{ Zona geográfica } & Antioquia y viejo caldas & 187 & $88,2 \%$ & 25 & $11,8 \%$ \\
& Central & 478 & $85,4 \%$ & 82 & $14,6 \%$ \\
& Costa & 251 & $90,9 \%$ & 25 & $9,1 \%$ \\
& Occidente & 221 & $87,7 \%$ & 31 & $12,3 \%$ \\
& Oriente & 285 & $90,2 \%$ & 31 & $9,8 \%$ \\
\hline
\end{tabular}


Con respecto a la intención que reportan los estudiantes de reaccionar cuando son testigos de que sus compañeros son objeto de intimidación (Tabla 6), se encontró que la gran mayoría dicen que reaccionarían ante este tipo de prácticas. Sin embargo, este resultado es contradictorio con las conductas descritas en la tabla 3. No se observan diferencias en función de las variables semestre, sexo ni zona geográfica (Taba 6).

Por último, en relación con las actitudes de los estudiantes de medicina hacia la práctica de intimidación, se encontró que los estudiantes de de II a V semestre presentan una actitud más favorables hacia este tipo de prácticas, que quienes están de VI a X. Así mismo los hombres tienen una actitud mas positiva que las mujeres. En cuanto a la zona geográfica, no se encuentran diferencias significativas.

\section{Discusión}

En la última década se han llevado a cabo diversos estudios sobre la presencia del "bullying" en las escuelas primaria y secundaria, ya que se considera que es en ésta etapa de formación cuando ocurre el fenómeno de la intimidación. Sin embargo, a través de los años los investigadores han logrado describir la presencia de "bullying" en los sitios de trabajo (49-51), estableciendo que éste puede existir en otros ámbitos como el de la academia. En la literatura científica hay pocos reportes sobre la intimidación en la universidad, desconociendo así las implicaciones que tiene para las víctimas la continuación de este tipo de violencia a lo largo del proceso de formación (colegio, universidad y trabajo).

Los resultados observados en este estudio confirma la presencia de episodios de intimidación en el ámbito de la educación superior y más específicamente, en los estudiantes de las escuelas de medicina del país, en donde se identificó en un $19,68 \%$ vr. un $30 \%$ de prevalencia del "bullying" en los colegios (26). Este dato es suficiente para dar una voz de alarma a las directivas, docentes y estudiantes y evidencian que este tipo de fenómenos no son un mito, sino una realidad que se está tomando los ambientes educativos de educación médica en Colombia.

A pesar de que en la mayoría de estudios realizados, la intimidación se presenta más en hombres que en mujeres, las diferencias encontradas en este estudio no fueron significativas entre ambos sexos, lo que podría interpretarse como la manifestación indistinta del "bullying" que se centra más específicamente en el tipo de intimidación ejercida. Así, las mujeres tienden realizar más un tipo de intimidación psicológica tipo exclusión o rechazo, mientras que los hombres suelen realizar matoneo verbal y físico. El tipo de "bullying" que predominó fue el verbal $(7,07 \%)$, con una notoria diferencia con el físico $(2,28 \%)$ y el rechazo $(3,08 \%)$ y casi sin presencia de "cyberbullying" $(0,44 \%)$. Estos resultados coinciden con el patrón reportado en otras investigaciones $(35,52,53)$ en cuanto a la forma sutil de manifestación de la intimidación, en las cuales se intenta mantener desapercibida la intimidación del otro a ojos de superiores. De igual manera, podría pensarse que los estudiantes no consideran que ciertas "trasgresiones" por medios virtuales sean intimidación, por lo que se sugiere indagar más sobre este aspecto entre los estudiantes en futuros estudios.

Retomando la concepción de comportamientos ejercidos por superiores como una forma posible de "bullying" dentro del ambiente médico, principalmente sobre estudiantes internos, se pudo evidenciar que: la intimidación ejercida por docentes $(10,68 \%)$ y compañeros $(10,10 \%)$ tuvo una gran diferencia con el que ejercen los especialistas y residentes $(4,17 \%)$, siendo los primeros (docentes) los victimarios más recurrentes. Estos datos permiten pensar que las "normas" y dinámicas de relación que se manejan en estos dos espacios tienen grandes diferencias, y que las relaciones de este último grupo (especialistas y residentes) podrían considerarse de menor subordinación que con los primeros. En cuanto al "bullying" ejercido por los docentes, en donde se observa el uso de la burla $(2,64 \%)$ y la humillación $(9,74 \%)$, deja una profunda preocupación que casi uno de cada diez docentes (por lo menos en el tema de la humillación) empleen denigración y la ofensa de la dignidad como una "estrategia" dentro su prácticas "pedagógicas" y como modelos de formación.

Al analizar las acciones tomadas por la víctima ante situaciones de intimidación, llama la atención el hecho de que $65,8 \%$ ignoran la situación, probablemente por temor a retaliaciones, sin pensar que esta estrategia se vuelve en contra de la víctima, en la medida en que permite al agresor continuar impunemente con su conducta. Sin embargo, al tener en cuenta que el $42,1 \%$ confronta a su agresor vr. un $6,5 \%$ que lo denuncia ante una autoridad académica, se esta dejando por sentado que la opción de tomar la justicia por sus mismas manos se considera una opción más legítima 
que las acciones respaldadas institucionalmente. Otra opción que la realiza uno de cada dos victimas del "bullyin" es contarle a un amigo, probablemente como una manera de buscar apoyo en alguien de la misma edad y grupo; sin embargo, es una medida que no permite disminuir la intimidación, pero si hacer propaganda del poder del agresor. Hoyos y col. (2009) también describen un patrón similar en su estudio, encontrando que las víctimas de matoneo cuentan en gran medida con el conocimiento de la situación por parte de un amigo/a y muy poca la posibilidad de hablar, de buscar ayuda por parte del decano u otra autoridad. Este último punto debe leerse a la luz del escenario en que se presenta, es decir en el ámbito de la educación superior que maneja otro tipo de dinámicas y procesos que los estudiados en colegios pero que independiente de ello, aboga por un sistema de quejas y consultas confiable y profesional. Es importante tener en cuenta que cuando no se hace nada ante el fenómeno de intimidación y mucho más, ante el denunciado, se envía está enviando el mensaje de que éste es aceptado (54).

Entre las condiciones para ser intimidado, sobresale la forma de pensar $(71,5 \%)$, seguida, por la apariencia física $(18,4 \%$ ) y la condición de ser hombre o mujer $(21,5 \%)$. Los estudios sobre "bullying" resaltan a las minorías, personas con rasgos excepcionales, género y desventajas económicas, como las causas más recurrentes en las características de las víctimas. En este caso particular, la forma de pensar permitiría evidenciar una coacción y el rechazo como formas más típicas de la violencia entre estudiantes, además de que ésta refleja más fácilmente diferencias culturales, religiosas o de habilidades mentales que son blanco fácil para los victimarios.

Por otra parte, el hecho que de dos a tres personas por cada diez estudiantes, tengan la percepción de que la práctica de matoneo sea algo que esté legitimado dentro del grupo social, hace pensar que probablemente el estudiante tienda a legitimizar y valorar como positivas esta formas de acción. Resulta preocupante, sobre todo en lo referente a la relación profesor estudiante, que entienda la humillación y la burla como una práctica "pedagógica" legitimada para exigir un buen comportamiento académico de sus estudiantes. Evidentemente, si el estudiante observa esta tipo de conductas en sus profesores, tenderá a repetirla en sus procesos de socialización con compañeros, pacientes y futuros colegas.
La intención de la mayoría de los estudiantes (por encima del 90\%) de reaccionar cuando son testigos de que alguien esta siento objeto de "bullying", contradice las prácticas que ellos realizan efectivamente cuando han sido testigos y la actitud positiva que tienen (al menos el $20 \%$ de ellos) hacia la intimidación. Por tanto la intención resulta ser un mal predictor de las conductas asertivas que los estudiantes deberían tener al momento de presenciar su realización. Este dato será de relevancia al momento de diseñar campañas preventivas, en donde los indicadores de los resultados de las mismas, deberán basarse sobre comportamiento público (búsqueda de ayuda, defensa de compañeros, etc.) y no sobre la conducta privada de las personas (pensamientos e intenciones).

En cuanto a las actitudes de los estudiantes hacia la intimidación, llama la atención el que los hombres tienen una actitud mucho más positiva que las mujeres, probablemente debido a que en nuestra sociedad las prácticas violentas son legítimas y son entendidas como una manera de ejercer el rol masculino, pero poco tiene que ver con el rol femenino. Por tanto, el enfoque de género en las prácticas de intimidación, es un elemento importante que debe ser tenido en cuenta para desarrollarse a la hora de trabajar el tema en las facultades de medicina.

Por otra parte, considerar los aspectos culturales, que de alguna manera podrían entreverse al realizar comparaciones de los resultados entre las distintas regiones del país, sería otro aspecto de relevancia a considerar a la hora de abordar la problemática. Se han llevado a cabo algunos estudios en diversas ciudades del país, pero no se tienen resultados por regiones de Colombia por lo cual es difícil comparar los valores obtenidos en el presente estudio y cualquier planteamiento que se haga serían sólo suposiciones. Los resultados de investigaciones recientes (55) han mostrado que los departamento de Amazonas, Casanare, Chocó, Cesar, Cundinamarca y Guaviare son los que más padecieron la intimidación. A pesar de que se cree que la intimidación es un fenómeno universal ya que ocurre indistintamente en todos los estratos, culturas y países, también hay quienes aseguran que las creencias y los valores culturales son importantes a la hora de explicar el maltrato entre iguales (11); por esta razón, las características de la región así como la situación de violencia general que se viva en el momento son un aspecto importante en la descripción de ésta situación. 
Esta investigación evidencia científicamente, que el "bullying" no es un mito sino una realidad que esta viva en las facultades de medicina, que probablemente tiene una vida reproductiva muy fructífera, y que por tanto debe ser un tema de preocupación para las facultades y hospitales, en la medida que perjudique la dinámica de relaciones interpersonales, la permanencia en la carrera, el rendimiento académico, la productividad e incluso la salud de los médicos y la de sus pacientes. Esto conlleva a la urgencia de desarrollar programas de intervención para la prevención y manejo de comportamientos de "bullying" en las facultades de medicina. Finalmente lo que deja como herencia esta investigación, son más interrogantes que respuestas. Por ejemplo, por mencionar algunas: ¿cuáles son determinantes del bullying?, ¿qué papel juega el rol de género en su origen y perpetuación?, ¿qué relación podría encontrarse entre el proceso de humanización de la medicina y la práctica de intimidación en sus centros de formación?, ¿̇de qué manera la práctica de intimidación puede afectar el proceso de enseñanza y aprendizaje?, ¿qué efectos sobre la formación y el aprendizaje tiene ser objeto de matoneo?, ¿qué relación existe entre la intimidación y otros tipos de prácticas violentas?, etc.

\section{Agradecimientos}

Los autores agradecen a la Universidad Militar Nueva Granada por la financiación del proyecto MED -400, a ASCOFAME y los decanos de todas las facultades de medicina que participaron en el estudio, a quienes no mencionamos por guardar la confidencialidad a la que nos comprometimos.

\section{Referencias}

1. Petersen A, Bleakley A, Brömer R, Marshall R. The Medical Humanities Today: Humane Health Care or Tool of Governance? J Med. Humanit. 2008; 29:1-4

2. Middleton J. Today I'm Grieving a Physician Suicide. Ann Fam Med 2008; 6 (3): 267-269.

3. Shanafelt T, West C, Zhao X, Novotny P, Kolars J, Habermann $\mathrm{T}$, Sloan J. Relationship Between Increased Personal Well-Being and Enhanced Empathy Among Internal Medicine Residents JGIM. 2005; 20 (7) , 559-564.

4. Castaño I, García M, Leguizamón L, Novoa M, Moreno I. Asociación entre el síndrome de estrés asistencial en residentes de medicina interna, el reporte de sus prácticas médicas de cuidados subóptimos y el reporte de los pacientes. Universitas Psychologica. 2006; 5 (3): 549-562.

5. Bellini L, Baime M, Shea J. Variation of Mood and Empathy During Internship. JAMA 2002;287(23):3143-3146

6. Olweus, D. Aggression in the schools: Bullies and whipping boys. Washington, DC: Hemisphere.1978

7. Instituto Nacional de Medicina Legal y Ciencias Forenses. Boletines y estadísticas: Lesiones Interpersonales. 2007
8. Lorenz, K. On aggression. New York: Harcourt, Brace \& World.1966

9. Heinemann P. Mobbing: Violencia de grupo por niños y adultos. Stockholm: Natur och kultur, 1972.

10. Olweus D. Bully/victim problems in school: Facts and intervention. EJPE. 1997; 12(4), 495-510.

11. Avilés, J.M. "Bullying": el maltrato entre iguales. Agresores, víctimas y testigos en la escuela. Salamanca: Amarú ediciones. 2003.

12. Quing Li. Bullying in the New playgrund: A research into cyberbullying and cyber Victimization. AJET 2007; 23(4), 435-454.

13. Yildirim A, Yildirim D. Mobbing in the workplace by peers and managers: mobbing experienced by nurses working in healthcare facilities in Turkey and its effect on nurses. JCN. 2007; 16 (8):1444-1453.

14. Leymann H. Mobbing and psychological terror at workplaces. Violence Vict. 1990; 5: 119-126.

15. Moreno B, Rodríguez A, Garrosa E, Morante M. Antecedentes organizacionales del acoso psicológico en el trabajo: un estudio exploratorio. Psicothema. 2005; 17 (4): 627-632.

16. Peralta M. El acoso laboral - mobbing- perspectiva psicológica. RES 2004; 18: 111-122.

17. Hoel H. \& Salin D. Organisational antecedents of workplace "Bullying". En Einarsen, S.

18. Meseguer de Pedro M, Soler M, Sáez M, García M. Workplace Mobbing and Effects on Workers' Health. The Spanish J Psychol. 2008; 11 (1): 219-227

19. Juárez-García A, Hernández E, Ramírez J. Mobbing un riesgo psicosocial latente. ERGA.2005; 13(3): 153-160.

20. Pranjić N, Maleš-Bilić L, Beganlić A, Mustajbegović J. Mobbing, Stress, and Work Ability Index among Physicians in Bosnia and Herzegovina: Survey Study CMJ. 2006; 47(5): 750-758.

21. Glew G, Fan M, Katon W, Rivara, F. "Bullying" and School Safety. JP. 2008; 152 (1): $123-128$

22. Benítez JL y Justicia F (2006). El maltrato entre iguales: descripción y análisis del fenómeno.EJREP, 4, 81-93

23. Kivimaki M, Elovainio M, Vahtera J. Workplace "Bullying" and sickness absence in hospital staff. OEM. 2000;57:656-60.

24. Stebbing J, Powles T. Stress in the workplace amongst medical professionals. JPGM. 2007;53:83-4.

25. Newbury-Birch D, Kamali F. Psychological stress, anxiety, depression, job satisfaction and personality characteristics in pre-registration house officers. JPGM. 2001;77:109-11.

26. Revista Cambio. Matoneo en las aulas. Noviembre. No. 698.1825. 2006.

27. National Institutes of Health-NICHD. (2006). "Bullying" Statistics. Recuperado de: http://parentingteens.about.com/cs/ bullying/a/bullying.htm. Visitada el 22 de marzo de 2007

28. Curtis J, Bowen I, Reid A. You have no credibility: nursing students' experiences of horizontal violence. Nurse Educ Pract.2007; 7(3): 156-163.

29. Dellasega,C. Bullying Among Nurses. AJN. 2009; 109(1): 52-58.

30. Bloisi W, \& Hoel H. Abusive work practices and bullying among chefs: A review of the literature. IJHosMan. 2008; 27, 649-656.

31. Rautio A, Sunnari V, Nuutinen M, Laitala M. Mistreatment of university students most common during medical studies. BMC Medical Education. 2005; 5: 36.

32. Ostvik K, Rudmin F. "Bullying" and Hazing Among Norwegian Army Soldiers: Two Studies of Prevalence, Context, and Cognition. Mil. Psychol. 2001;13 (1): 17 - 39

33. Salin, D. "Bullying" and organizational politics in competitive and rapidly changing work environments. IJMDM. 2003; 4 (1): 335 - 46

34. Frank E, Carrera JS, Stratton T, Bickel J, Nora LM. Experiences of belittlement and harassment and their correlates among 
medical students in the United States: longitudinal survey. BMJ. 2006; 333: 682

35. Hoyos O, Romero L, Valega S y Molinares, C. El Maltrato entre Iguales por Abuso de Poder y Exclusión Social en Estudiantes de una Universidad Privada de la Ciudad de Barranquilla. Psykhé. 2009; 16: 109-125.

36. Wood D. "Bullying" and harrasment in medical schools. Student BMJ. 2006;333 (7570)664-665.

37. Quine L. Workplace "Bullying" in junior doctors: questionnaire survey. BMJ. 2002;324:878-879

38. Hicks B. Time to stop "Bullying" and intimidation. BJHM. 2000; 61 (6): 428-431.

39. Kassebaum D, Cutler E. On the culture of student abuse in medical school. AAMC. 1998;73(11):1149-58.

40. Frank E, Rothenberg R, Lewis Ch, Belodoff BF. Correlates of Physcian' Prevention-Related Practices. Findings From the Women Physians' Health Study. Arch. Fam Med. 2000;9: 359-67.

41. Sanabria P, Gonzalez L, Urrego D. Estilos de vida saludables en profesionales de la salud colombianos. Estudio exploratorio. Rev Med. 2007; 15 (2): 207-217.

42. Paredes O. Nuevos retos en la educación: el bienestar como una nueva competencia médica. Universitas Médicas 2008; 49 (1): 97-110.

43. Wallace J, Lemaire \& Ghali W. Physician wellness: a missing quality indicator. The Lance.t 2009; 374 (9702):1714-1721.

44. Haidet P, Stein H. The Role of the Student-Teacher Relationship in the Formation of Physicians. The Hidden Curriculum as Process. JGIM. 2006; 21 (1): S16-S20.

45. Lempp H, Seale C. The hidden curriculum in undergraduate medical education: qualitative study of medical students' perceptions of teaching. BMJ.2004;329(7469):770-3.
46. Fekkes M, Pijpers M, Verloove-Vanhorick S. Bullying: who does what, when and where? Involvement of children, teachers and parents in bullying behavior. Health Edu. on Res. 2005; 20(1):81-91.

47. Quine L.Workplace bullying injunior doctors: questionnaire survey.BMJ. 202;324(7342):8-9.

48. Cabezas C. Violencia escolar: El acoso del profesor hacia el alumno, 2008. Madrid. ISBN: 978-84-92539-19-2

49. Rodríguez-Carballeira A, Escartín J, Visauta B, Porrúa C, \& Martín-Peña J .Categorization and hierarchy of workplace bullying strategies: A Delphi survey. Spanish J psychol. 2010; 13, 297-308.

50. Scott J, Blanshard C, Child S. Workplace bullying of junior doctors: crosssectional questionnaire survey. NZ Med J. 2008 121: 10-4.

51. Paice E, Aitken M, Houghton A, Firth-Cozens J. Bullying among doctors in training: cross sectional questionnaire survey. BMJ. 2004;329:658-9.

52. Reátiga María E. Los recuerdos del maltrato entre compañeros en la vida escolar. Psicología desde el caribe. 2009; 132147.

53. Lima Braga L, Lisboa C. Estratégias de Coping para Lidar com o Processo de Bullying:Um Estudo Qualitativo. IJP. 2010; 44(2):321-331

54. Elliott M \& Kilpatrick J. How to Stop Bullying: A Kidscape Training Guide. Kidscape, 2002. London.

55. Chaux, E. Agresión y Competencias Ciudadanas en Colombia: Análisis preliminar con base en la Prueba Nacional ICFESSABER de Competencias Ciudadanas. Taller Internacional de Formación en Competencias Ciudadanas. Organización de Estados Americanos. Bogotá. 2005. 